\title{
Multiple Field-Induced Phase Transitions in the Geometrically Frustrated Dipolar Magnet: $\mathrm{Gd}_{2} \mathrm{Ti}_{2} \mathrm{O}_{7}$
}

\author{
A. P. Ramirez, ${ }^{1, *}$ B. S. Shastry, ${ }^{2,3,5}$ A. Hayashi, ${ }^{4}$ J. J. Krajewski, ${ }^{3}$ D. A. Huse, ${ }^{5}$ and R. J. Cava ${ }^{4}$ \\ ${ }^{1}$ Los Alamos National Laboratory, K764, Los Alamos, New Mexico 87545 \\ ${ }^{2}$ Department of Physics, Indian Institute of Science, Bangalore 560012, India \\ ${ }^{3}$ Bell Laboratories, Lucent Technologies, 600 Mountain Avenue, Murray Hill, New Jersey 07974 \\ ${ }^{4}$ Chemistry Department, Princeton University, Princeton, New Jersey 08540 \\ ${ }^{5}$ Physics Department, Princeton University, Princeton, New Jersey 08540
}

(Received 6 August 2001; published 23 July 2002)

\begin{abstract}
Field-driven phase transitions generally arise from competition between Zeeman energy and exchange or crystal-field anisotropy. Here we present the phase diagram of a frustrated pyrochlore magnet $\mathrm{Gd}_{2} \mathrm{Ti}_{2} \mathrm{O}_{7}$, where crystal-field splitting is small compared to the dipolar energy. We find good agreement between zero-temperature critical fields and those obtained from a mean-field model. Here, dipolar interactions couple real space and spin space, so the transitions in $\mathrm{Gd}_{2} \mathrm{Ti}_{2} \mathrm{O}_{7}$ arise from field-induced "cooperative anisotropy," reflecting the broken spatial symmetries of the pyrochlore lattice.
\end{abstract}

DOI: $10.1103 /$ PhysRevLett.89.067202

PACS numbers: 75.30.Kz, 75.10.-b, 75.50.Ee

The pyrochlore lattice, which consists of corner-shared tetrahedra of spins, has special significance in the study of geometrically frustrated systems. The tetrahedron forms a natural basis of a four-sublattice antiferromagnet. However, corner sharing and the resulting Maxwellian underconstraint reduce the role of intertetrahedral correlations [1]. This balance of local and extended degrees of freedom has led to a variety of unusual collective behaviors, including "cooperative paramagnetism" [2,3], ultrasmall disorder spin glass [4], and spin ice freezing [5,6]. Of fundamental interest is the interplay between frustrating interactions and applied field. In the Ising spin ice system, $\mathrm{Dy}_{2} \mathrm{Ti}_{2} \mathrm{O}_{7}$, a magnetic field lifts the tetrahedral degeneracy, inducing antiferromagnetic long-range order in a system which was disordered in zero field [6].

In this Letter, we present the $H-T$ phase diagram for a dipolar Heisenberg pyrochlore magnet $\mathrm{Gd}_{2} \mathrm{Ti}_{2} \mathrm{O}_{7}$ as determined by specific heat $C(T)$ and ac susceptibility $\chi(T)$. $\mathrm{Gd}_{2} \mathrm{Ti}_{2} \mathrm{O}_{7}$ has a Weiss constant $\theta_{\mathrm{W}}$ of $\sim 10 \mathrm{~K}$ [7] and a spin-freezing transition $T_{c}$ around $1 \mathrm{~K}$, and thus $f \equiv$ $\theta_{\mathrm{W}} / T_{c} \cong 10$. We find here an unusually complex phase diagram for a Heisenberg system. Agreement between critical field values and mean-field theory suggests a novel origin for this complexity - the mapping of broken spatial symmetries of the pyrochlore lattice onto spin space.

Two different types of $\mathrm{Gd}_{2} \mathrm{Ti}_{2} \mathrm{O}_{7}$ samples were studied. Polycrystalline samples of $\mathrm{Gd}_{2} \mathrm{Ti}_{2} \mathrm{O}_{7}$ were synthesized from high purity $\mathrm{Gd}_{2} \mathrm{O}_{3}$ and $\mathrm{TiO}_{2}$ powders, mixed in the stoichiometric ratio, and heated in air in pristine, highdensity $\mathrm{Al}_{2} \mathrm{O}_{3}$ crucibles. For the polycrystalline materials, initial heating was at $1200^{\circ} \mathrm{C}$ for $15 \mathrm{~h}$. Subsequent heatings were at $1350{ }^{\circ} \mathrm{C}$ for $60 \mathrm{~h}$, with several intermediate grindings. The final treatment was at $1100^{\circ} \mathrm{C}$ for $24 \mathrm{~h}$ in $\mathrm{O}_{2}$. The resultant material was single phase by powder $\mathrm{x}-$ ray diffraction. Single crystals were obtained by melting single phase powder under Ar, and then annealing for $24 \mathrm{~h}$ in $\mathrm{O}_{2}$ at $1100{ }^{\circ} \mathrm{C}$. Measurements of the weight loss on heating our single phase polycrystalline $\mathrm{Gd}_{2} \mathrm{Ti}_{2} \mathrm{O}_{7}$ in a thermogravimetric analysis in a variety of atmospheres at temperatures up to $1100{ }^{\circ} \mathrm{C}$ indicate that there is no significant nonstoichiometry in the oxygen content under the synthetic conditions employed. In all samples chosen for this study, the narrow diffraction peak widths indicated excellent crystallinity, and the refined crystallographic cell parameters were identical within the precision of conventional powder $\mathrm{x}$-ray diffraction. Our low-temperature results are different from those previously reported [7]. However, it has been reported that high-temperature synthesis can lead to off-stoichiometry [8] in the Gd to Ti ratio for this compound, in turn leading to structural disorder. The long synthetic times, as well as the low-temperature oxygen anneal, minimize the disorder and nonstoichiometry for the present samples. Structural disorder, possibly present due to the short reaction time or lack of a lowtemperature anneal, may be responsible for the suppression in previous work [7] of some of the transitions observed here.

For $T>2 \mathrm{~K}$, the dc susceptibility was measured with a commercial magnetometer. Below $1.5 \mathrm{~K}$, ac susceptibility was measured using a mutual inductance coil technique operating at $143 \mathrm{~Hz}$. For this measurement, the powder was mixed with Stycast 1266 epoxy and cast with an embedded matrix of fine $\mathrm{Cu}$ wires for thermal equilibration. The specific heat was measured using a standard semiadiabatic heat-pulse technique. The powder samples were cold sintered with fine $\mathrm{Ag}$ powder to aid thermal equilibration.

In Fig. 1 we show $\chi(T)$ above $2 \mathrm{~K}$. Fitting the data to a straight line above $25 \mathrm{~K}$ yields a value for the effective moment of $7.98 \mu_{\mathrm{B}}\left(\theta_{\mathrm{W}}=-9.4 \mathrm{~K}\right)$ in good agreement with the $S=7 / 2$ free-ion value of $7.94 \mu_{\mathrm{B}}$ and previous work [7]. In the lower inset in Fig. 1 we show a $C(T)$ measurement for one of our samples (powder 1). 


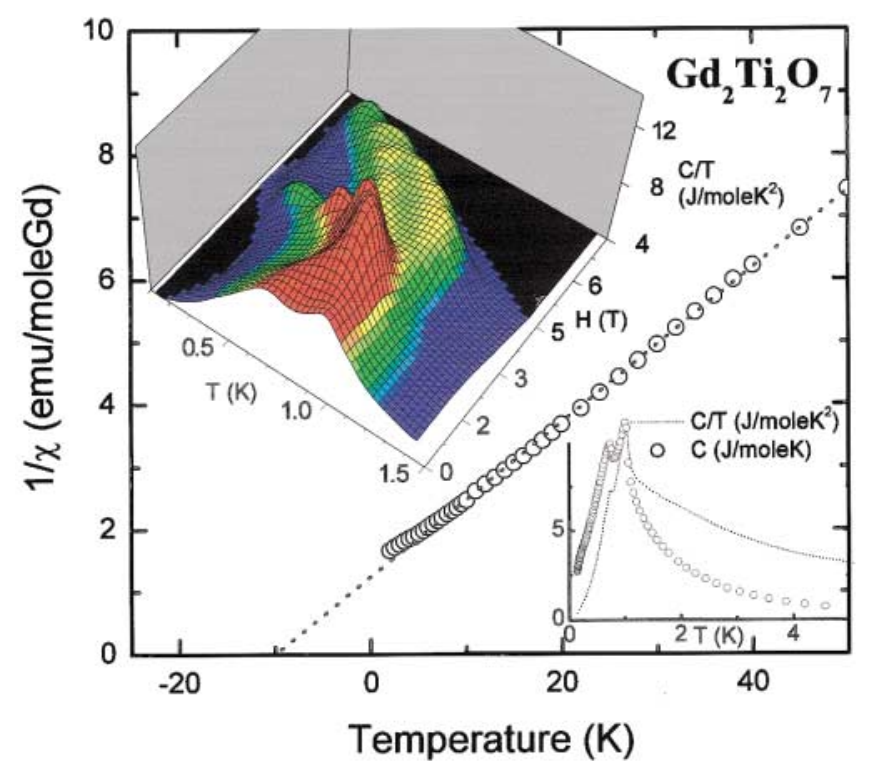

FIG. 1 (color). Inverse susceptibility $1 / \chi(T)$ versus temperature for $\mathrm{Gd}_{2} \mathrm{Ti}_{2} \mathrm{O}_{7}$. The dashed line is to guide the eye. The upper inset shows the specific heat divided by temperature, $C(T) / T$ in the full temperature and field range of this work. The lower inset shows the specific heat $C(T)$ as well as $C(T) / T$ for $\mathrm{Gd}_{2} \mathrm{Ti}_{2} \mathrm{O}_{7}$ over a large temperature scale.

Integrating the data up to $5 \mathrm{~K}$ yields an entropy of $92 \%$ of the full $R \ln 8$ expected for $\mathrm{Gd}^{3+}$, consistent with the expected small single-ion anisotropy. In Fig. 2 we show $C(T) / T$ below $1.5 \mathrm{~K}$ at $H=0$ for three different samples, demonstrating the reproducibility of the multiple zero-field

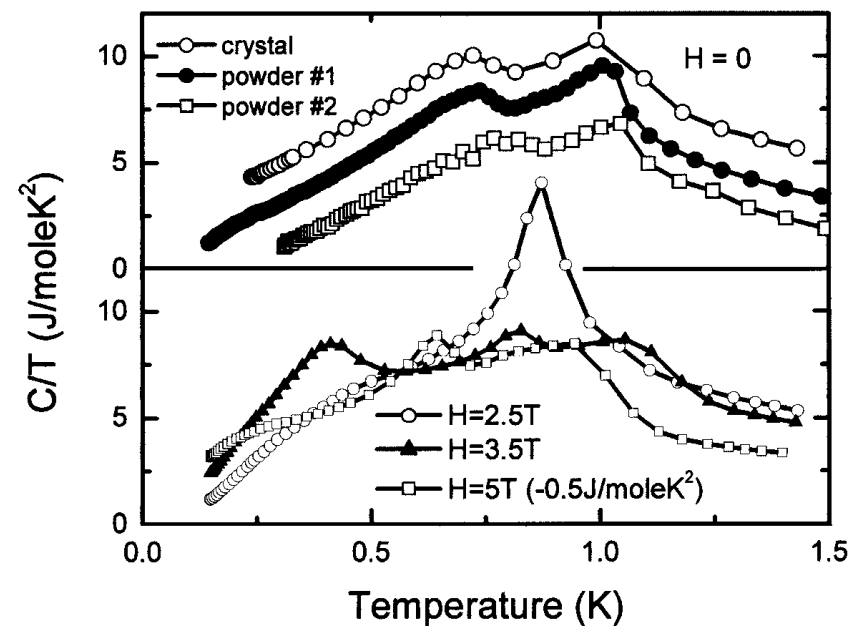

FIG. 2. Specific heat divided by temperature $C(T) / T$ for the crystallites sample of $\mathrm{Gd}_{2} \mathrm{Ti}_{2} \mathrm{O}_{7}$ at different values of applied field. The upper panel also shows $C(T) / T$ at $H=0$ for three different samples, two prepared by solid state synthesis and one prepared by arc melting and consisting of large $(0.1 \mathrm{~mm})$ crystallites. The powder \#1 and powder \#2 data are offset by 1.5 and $3.0 \mathrm{~J} / \mathrm{mole}^{2}$, respectively, for clarity. The lower panel shows the $C(T) / T$ data in a few finite fields. transitions. In Fig. 2 is also shown $C(T) / T$ for the crystallite sample at a few different field values, demonstrating the merging of the zero-field peaks, followed by splitting at higher fields. Figure 1 (inset) is a surface plot of $C(T) / T$, showing the full range of data [here, $C(T)$ was measured every $0.5 \mathrm{~T}$ up to $8 \mathrm{~T}$ ]. Similar data were obtained for a powder sample.

ac-susceptibility, $\chi_{\mathrm{ac}}(H)$, measurements as shown in Fig. 3 complement the $C(T)$ data in their greater sensitivity to high-field phase boundaries, and the full phase diagram is shown in Fig. 4. Multiple field-induced phase transitions are by themselves not unusual, usually seen in systems with uniaxial anisotropy, and result from near degeneracy of phases with different wave vector or symmetry [9]. The behavior in $\mathrm{Gd}_{2} \mathrm{Ti}_{2} \mathrm{O}_{7}$ is, however, markedly different from other such systems, since $\mathrm{Gd}^{3+}$ does not typically have significant uniaxial anisotropy. Rather, the behavior is a counterpart to that seen in $\mathrm{Gd}_{3} \mathrm{Ga}_{5} \mathrm{O}_{12}$, which, while fully frustrated for $H=0$, also exhibits sharp field-induced critical behavior [10].

Previous zero-field theory on Heisenberg pyrochlores showed that by including long-range superexchange [7] or dipole-dipole coupling [11], an ordered phase is stabilized at low temperature. At zero temperature six distinct ground states are found within a simple four-sublattice model, and the evolution of these ground states in a field provides features rather similar to our data, as we show below. Since the measurements were made on a polycrystalline sample, the multiple phase transitions as the field is increased may be from crystallites of different orientation. Our model shows either one or two low-temperature phase transitions with increasing field for each of the highsymmetry orientations of the field. The inclusion of dipolar interactions is a crucial feature of this model and should be compared with recent theoretical work on the phase



FIG. 3 (color). ac susceptibility $\chi_{\text {ac }}$ for $\mathrm{Gd}_{2} \mathrm{Ti}_{2} \mathrm{O}_{7}$ as a function of field at fixed temperatures. The inset shows the full $\chi_{\text {ac }}$ data set for two different temperatures. 
diagram of the frustrated Heisenberg square-lattice antiferromagnet [12], which bears some similarity to our system, but without dipolar interactions.

We set up a simple model in the spirit of models used to explain the spin-flop transition in uniaxial materials such as $\mathrm{MnF}_{2}$. The model incorporates a dipolar interaction in addition to the superexchange. A mean-field treatment of the dipolar interaction [13] yields the phenomenological anisotropy energy. Unlike the case of $\mathrm{MnF}_{2}$, which has uniaxial symmetry in a two-sublattice antiferromagnet, the present case corresponds to cubic symmetry in a foursublattice magnet. We describe the model and its consequences only qualitatively below, reserving a detailed exposition for a future publication.

In our model, we locate spins on the pyrochlore lattice, viewed as a fcc lattice [primitive vectors $a / 2(0,1,1)$, $a / 2(1,0,1), a / 2(1,1,0)]$, with four basis vectors $[0$, $a / 4(0,1,1), a / 4(1,0,1), a / 4(1,1,0)]$ defining the four sublattices. We replace each spin on sublattice $j$ by $S \vec{\eta}(j)$ and seek to determine the directions of the unit vectors $\vec{\eta}(j)$. The problem then reduces to that of just four classical vectors, and the energy is

$$
\begin{aligned}
4 F / N= & +J S^{2} \sum_{j, k}^{\prime} \vec{\eta}(j) \cdot \vec{\eta}(k)-g \mu_{\mathrm{B}} \sum_{j} \vec{\eta}(j) \cdot \vec{B} \\
& +\left(g \mu_{\mathrm{B}} S\right)^{2} /\left(2 a^{3}\right) \sum_{i \neq j} \sum_{a, b=x, y, z} \eta^{a}(i) D^{a b}(i, j) \eta^{b}(j),
\end{aligned}
$$

where $g=7 / 2$ and $J$ is the superexchange energy. The dimensionless matrix $D^{a b}$ represents the dipolar energy. This matrix is determined by summing over a sufficiently large spherical region (the Lorenz sphere); evaluation of terms out to a distance of $\sim 10^{2}$ sites provides a wellconverged result. A version of this problem was considered by Palmer and Chalker [11], who found that in the absence of an external $B$ field this model has six ground states. We find for $B=0$ precisely the same ground states and describe these more fully next.

In our treatment, the only adjustable parameter is $J$ and we found fair agreement with the experimental phase boundaries for $J=0.4 \mathrm{~K}$. With this value for $J$, and $a=$ $10 \AA$, we find the exchange energy $J \sim 0.60 E_{d}$, where $E_{d}=16 \sqrt{2}\left(g \mu_{\mathrm{B}} S\right)^{2} / a^{3} \sim 0.653 \mathrm{~K}$ is the dipolar energy for a pair of nearest-neighbor moments. We found the following ground states for $B=0$ (in each pattern $\langle i j\rangle$, the spins are aligned in the $i j$ plane):

$$
\begin{aligned}
& \text { Pattern }\langle\mathbf{x y}\rangle: \vec{\eta}(1)=\frac{1}{2}\{1,-1,0\}, \quad \vec{\eta}(2)=\frac{1}{2}\{-1,-1,0\}, \quad \vec{\eta}(3)=\frac{1}{2}\{1,1,0\}, \quad \vec{\eta}(4)=\frac{1}{2}\{-1,1,0\} . \\
& \text { Pattern }\langle\mathbf{x z}\rangle: \vec{\eta}(1)=\frac{1}{2}\{-1,0,1\}, \quad \vec{\eta}(2)=\frac{1}{2}\{1,0,1\}, \vec{\eta}(3)=\frac{1}{2}\{1,0,-1\}, \quad \vec{\eta}(4)=\frac{1}{2}\{-1,0,-1\} . \\
& \text { Pattern }\langle\mathbf{y z}\rangle: \vec{\eta}(1)=\frac{1}{2}\{0,1,-1\}, \quad \vec{\eta}(2)=\frac{1}{2}\{0,-1,1\}, \quad \vec{\eta}(3)=\frac{1}{2}\{0,-1,-1\}, \quad \vec{\eta}(4)=\frac{1}{2}\{0,1,1\} .
\end{aligned}
$$

These patterns and their fully reversed conjugates, e.g., $\langle\mathbf{x y}\rangle$, are evolved in the presence of an external field oriented in various directions and increased in small steps, using standard minimization procedures, we denote the resultants as $\langle\mathbf{x y}\rangle_{h},\langle\mathbf{x y}\rangle_{h}$, etc.

In order to study the stability of the mean-field states, we compute the frequencies of the antiferromagnetic magnons at zero wave number. We linearize the dynamical equations of motion around a mean-field solution and obtain a dynamical matrix for transverse fluctuations. We also compute the Hessian matrix of stability for each mean-field solution (Fig. 4) by considering variations $\vec{\eta}(j) \rightarrow \vec{\eta}(j)+$ $\vec{\delta}(j)$. The vector $\vec{\delta}$ is orthogonal to $\vec{\eta}(j)$; the energy is expanded out to quadratic terms in the variation giving the Hessian matrix. Finally, we calculate the (bilinear) tensor "order parameters" $T^{a b}=\frac{1}{4} \sum_{j=1}^{4} \eta^{a}(j) \eta^{b}$ (j) (Fig. 4).

The lattice symmetry that is broken when the magnetic field is along $\langle 110\rangle$ is a reflection in the plane spanned by the field and the $z$ axis. The four states $\langle\mathbf{x z}\rangle_{h},\langle\mathbf{y z}\rangle_{h},\langle\mathbf{x z}\rangle_{h}$, and $\langle\mathbf{y z}\rangle_{h}$ are energetically degenerate and thermodynamically stable at small field. States $\langle\mathbf{x y}\rangle_{h}$ and $\langle\mathbf{x y}\rangle_{h}$ have higher energies. The four low-field states all have a transition at $B \sim 4 \mathrm{~T}$, characterized by a deep minimum in the lowest magnon frequency, as well as in the lowest Hessian eigenvalue. At this $4 \mathrm{~T}$ transition the pairs of states that transform into one another under the reflection operation merge pairwise, with $\langle\mathbf{x z}\rangle_{h}$ and $\langle\mathbf{y z}\rangle_{h}$ becoming the same state and $\langle\mathbf{y z}\rangle_{h}$ and $\langle\mathbf{x z}\rangle_{h}$ also merging. The two remaining states are degenerate, but not related by any obvious symmetry of the system. Another transition where these two remaining states become the same occurs at $B \sim 7 \mathrm{~T}$, again with a vanishing of the lowest magnon and Hessian eigenvalue. Beyond $7 \mathrm{~T}$ there is only a unique paramagnetic state. Thus both of the transitions are Ising-like, with pairs of states merging.

For a field along the $\langle 100\rangle$ direction, the linear magnetization $M^{x}$ exhibits no obvious singularities as a function of field. However, there are clear indications of phase transitions in the bilinear order parameters $T^{i j}$. It is perhaps important to point out that similar bilinear operators are the primary order parameters in nematic liquid crystals. As we increase $B$, the six principal states remain degenerate until a critical field of $B \sim 6 \mathrm{~T}$. The relevant symmetry here is rotation about this fourfold axis. States $\langle\mathbf{y z}\rangle_{h}$ and $\langle\mathbf{y z}\rangle_{h}$ transform into one another under the symmetry operation consisting of a $90^{\circ}$ rotation about $\langle 100\rangle$ and a translation. At $6 \mathrm{~T}$ there is a transition to just two states with the 


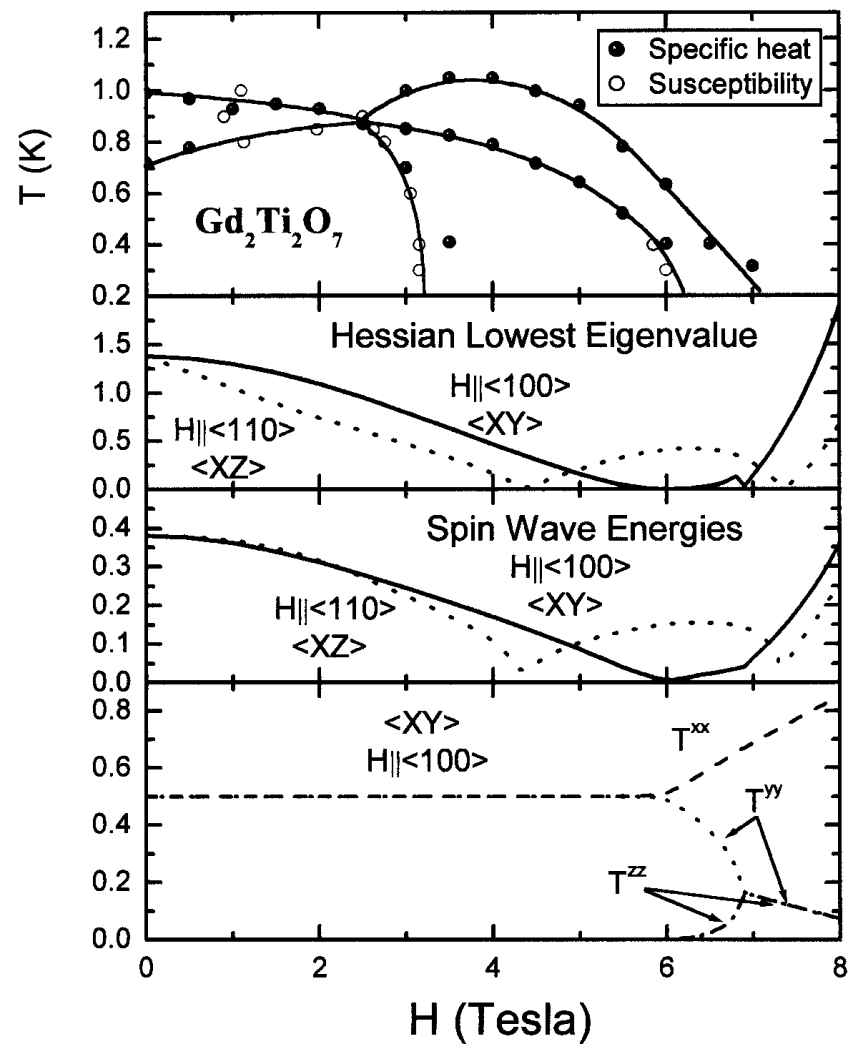

FIG. 4. Upper panel: Phase diagram for $\mathrm{Gd}_{2} \mathrm{Ti}_{2} \mathrm{O}_{7}$. The different symbols denote the position of peaks in either $C(T) / T$ or $\chi_{\text {ac }}(H)$. Second panel: Lowest Hessian eigenvalues as a function of field for two principal directions. Third panel: Lowest spinwave energies as a function of field for two principal directions. Bottom panel: The bilinear order parameters $T^{i i}$ for the $\langle\mathbf{x y}\rangle$ state as a function of field along the $\langle 100\rangle$ direction.

coming together of $\langle\mathbf{x y}\rangle_{h},\langle\mathbf{y z}\rangle_{h},\langle\mathbf{x y}\rangle_{h}$, and the other trio of states. This restores the symmetry under $180^{\circ}$ rotation around $\langle 100\rangle$. The two remaining states between 6 and $7 \mathrm{~T}$ transform into one another under the combined $90^{\circ}$ rotation and translation. At $\sim 7 \mathrm{~T}$ this symmetry is restored in apparent Ising-like transition.

Note that for the field along $\langle 111\rangle$ there is only the one transition, where both rotation and reflection symmetries are broken simultaneously, and here there are no extra degeneracies that arise from something other than the lattice symmetries.

Thus we see that among the principal directions a meanfield theory reproduces the number of transitions and approximate locations of the three observed critical fields. The transitions occur as the result of the collapse of degeneracies among states that in many, but not all, cases are symmetry related, as summarized in Fig. 5. The surprise here is the high degeneracy that survives in rather large magnetic fields - a situation we believe is uniquely caused by dipolar spins in a geometrically frustrated configuration. Thus we have a situation reminiscent of little groups [14] which form as a result of high symmetry along particular crystallographic directions. Here, however, the high

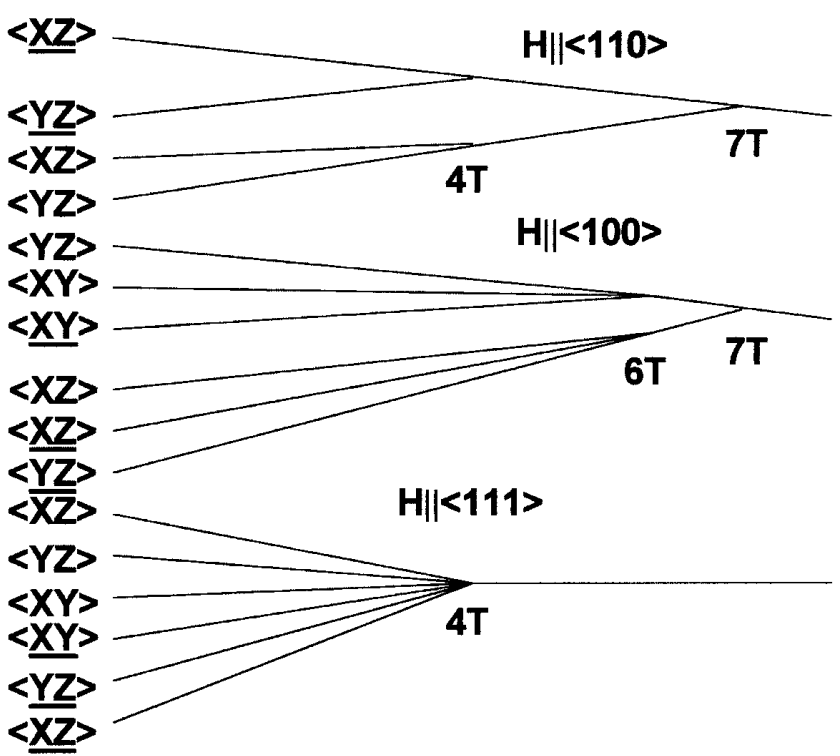

FIG. 5. Evolution and branching of the degenerate phases of the dipolar pyrochlore system as a function of field in the principal directions. Note the vertical direction is not an energy axis.

symmetry results from complex frustrated interactions rather than from a particular crystalline anisotropy.

In summary, we have shown that the geometrically frustrated dipolar Heisenberg magnet $\mathrm{Gd}_{2} \mathrm{Ti}_{2} \mathrm{O}_{7}$ has a complex phase diagram. A minimal mean-field model is presented which produces the low-temperature critical field values. Further experimental work, in particular, ESR and polarized neutron scattering, preferably using single crystals, is needed to test our proposed simple model for the various phase transitions.

*Experimental work performed at Bell Laboratories.

[1] R. Moessner and J. T. Chalker, Phys. Rev. B 58, 12049 (1998).

[2] J. Villain, Z. Phys. B 33, 31 (1979).

[3] J. S. Gardner et al., Phys. Rev. Lett. 82, 1012 (1999).

[4] J.E. Greedan et al., Solid State Commun. 59, 895 (1986).

[5] M. J. Harris et al., Phys. Rev. Lett. 79, 2554 (1997).

[6] A. P. Ramirez et al., Nature (London) 399, 333 (1999).

[7] N.P. Raju et al., Phys. Rev. B 59, 14489 (1999).

[8] J. L. Waring and S. J. Schneider, J. Res. Natl. Bur. Stand. Sect. A 69, 255 (1965).

[9] E. Stryjewski and N. Giordano, Adv. Phys. 26, 487 (1977).

[10] P. Schiffer et al., Phys. Rev. Lett. 73, 2500 (1994).

[11] S.E. Palmer and J.T. Chalker, Phys. Rev. B 62, 488 (2000).

[12] M.E. Zhitomirsky, A. Honecker, and O.A. Petrenko, Phys. Rev. Lett. 85, 3269 (2000).

[13] F. Keffer, in Encyclopedia of Physics (Springer-Verlag, Berlin, 1966), Vol. 18.

[14] M. Hamermesh, Group Theory and its Application to Physical Problems (Addison-Wesley, Reading, MA, 1962). 